\title{
Addressing unwarranted clinical variation in healthcare as a quality improvement process
}

\author{
Tobias Hodgson \\ The University of Queensland \\ t.hodgson@business.uq.edu.au
}

\author{
Andrew Burton-Jones \\ The University of Queensland \\ abj@business.uq.edu.au
}

\author{
Clair Sullivan \\ The University of Queensland \\ c.sullivan1@uq.edu.au
}

\begin{abstract}
The reduction of unwarranted clinical variation is a common goal of healthcare systems worldwide. However, the process of developing and implementing variation reducing interventions as a quality improvement process is often overlooked and performed sub-optimally within healthcare organizations. This gap in practice is mirrored by a gap in existing research.

The development of a clinical variation specific prescriptive model will assist with the identification, development and application of healthcare specific variation reducing initiatives. Such a model should complement the existing plan, do, study, act quality (PDSA) improvement methodology and respect the learning health systems (LHS) learning cycle.

Development through the lens of the quadruple aim of healthcare will ensure that the focus remains true to the core values of clinical organizations.

Addressing unwarranted clinical variation is a complex task, however. With organizational support, the utilization of collaborative methodologies and the leveraging of available digital health technologies, healthcare organizations are provided the greatest opportunity for the reduction of unwarranted clinical variation and the optimization of healthcare outcomes.
\end{abstract}

\section{Introduction}

The reduction of unwarranted clinical variation is a common goal of healthcare systems worldwide. While not all clinical variation is negative, and instead may facilitate beneficial change, variation is unwarranted when it is not justified by clinical imperatives, patient needs/preferences, or innovation.

The reduction of unwarranted clinical variation may provide benefits that satisfy the quadruple aim of healthcare: to improve patient care, population health, cost of care, and clinician experience $[1,2]$.

Addressing unwarranted clinical variation is a high priority complex task involving multiple elements with intricate interactions occurring between these components. Trade-offs often exist between the potentially competing elements of cost, quality, population health, clinicians, and patients. How to best identify, understand and address these trade-offs is an area that deserves additional attention as often the improvement of one aspect may be detrimental to another. For example, reduced costs via reductions in tests/drugs may have an adverse effect on patient outcomes.

Clinical variation is often addressed by healthcare organizations with the standardization of clinical practice via quality improvement (QI) initiatives, ranging from process control (guidelines and pathways) to design development efforts (systems) [3, 4].

Electronic medical/health record $\left(\mathrm{EMR} / \mathrm{EHR}^{1}\right)$ systems provide organizations the opportunity to identify variation. Once identified, they can implement variation reducing QI initiatives by utilizing the everincreasing number of functions and features available in digital health systems. While this has been achieved with limited success ad-hoc, identifying the low-level details required to effectively select and implement unwarranted clinical variation reduction QI initiatives is a priority [5-7]. This work is predicated by literature review undertaken by the authors examining the impact of EMRs on variation in clinical care processes in hospital settings [6].

The objective of this paper is to introduce a model beneficial to healthcare organizations developing or implementing unwarranted clinical variation initiatives.

\section{Theoretical framework}

The healthcare sector has adopted numerous QI methodologies from clinical audit, Lean/Six sigma, plan do study/check act (PDSA/PDCA), process mapping, statistical process control, total quality management (TQM), root cause analysis and beyond with varying levels of success [8-10]. The majority of which center around a formal structure of continued iterative development undertaken by an organization.

\footnotetext{
${ }^{1}$ Electronic health record (EHR) \& electronic medical record (EMR) may be used interchangeably and will be referred to as EMR throughout this paper.
} 
These quality improvement cycles often have similar stages of planning, doing/implementing, measuring/studying and act/sustaining improvements within their models [9, 11]. Perhaps the best-known methodology is PDSA.

PDSA is a simple framework which has the advantage of being able to support many lower-level complex approaches such as organizational, social interventions to more technical computational based process modelling such CRISP-DM [12].

The PDSA cycle's origins began with the Shewhart and later the Deming cycles and remains among the leading QI components worldwide [8, 13-15]. While the PDSA cycle is easily understood and applicable to most industries, applying this normative model to the realworld task of addressing a specific instance of clinical variation is not easily achieved.

To this end, a model that specifically address the requirements of the clinical variation reduction process has been developed to complement the existing PDSA methodology and the requirements of a learning health system (LHS) (Figure 1).

Figure 1: Unwarranted clinical variation reduction process

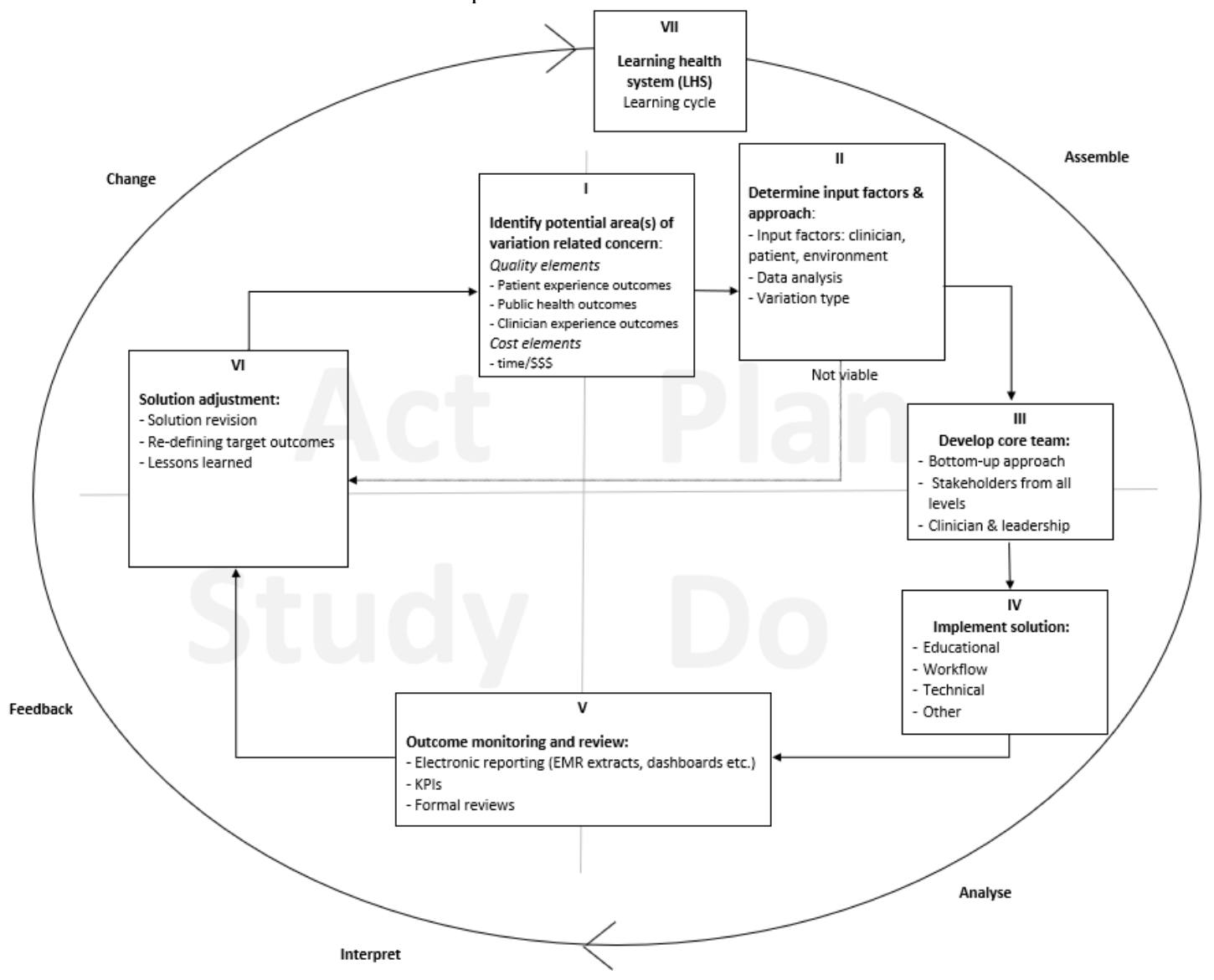

\subsection{Variation identification}

(Figure 1-I)

The identification of unwarranted clinical variation is an intricate task as it involves differentiating the clinical practice of clinicians with the intervention (such as the guidelines/pathway) from those outcomes associated with the intervention, along with the clinician use of the intervention. This may be best achieved through the lens of the quadruple aim of healthcare [1].
By assessing outcomes and processes against the quadruple aim criteria, deficits and variations within the existing system are easily identifiable, thus minimizing unjustified differences between healthcare processes or outcomes when compared to peers, or to a gold standard is the ultimate goal [16].

Information technology (IT) advancements, digital health, and the use of clinical information systems (CIS) and EMRs have transformed clinical monitoring practices and provided the ability to more easily identify 
and demonstrate elements of clinical variation (often in real-time). This may be achieved with the use of alerts, clinical decision support systems (CDSS), dashboard summaries, and big data analytics [17-19].

Traditional methods of variation identification should continue to be utilized in conjunction with digital based techniques. These may include anecdotal evidence from expert stakeholders, bedside reviews/rounds, financial analysis, clinician surveys, peak organization/network evidence and trend analysis such as statistical process control charts [20,21].

\subsection{Process input factors}

(Figure 1-II)

Once the targeted aspect of clinical variation has been identified, the process of input factors discovery may commence. These factors may be supply-side with the clinician, demand-side factors from the consumer/patient or contextual environmental factors [22]:

- Clinician factors (supply-side): expertise, training and experience, preference, practice style.

- Consumer factors (demand-side): case complexity, consumer preference, social determinants of health.

- Environmental factors (context): local guidelines, available resources, hospital casemix.

In addition to affecting clinical practice in isolation, these individual factors may combine to influence clinical variation as a collective [22]. Determining the appropriate (or allowable) degree of variation for any specific clinical practice is a task typically undertaken at a local hospital or health service level to ensure that any specific district conditions are accounted for.

That said, it is essential that the element of unwarranted clinical variation to be addressed meets some fundamental entry criteria. The variation should be deemed unwarranted, should align with an area of value for the organization (often cost linked), align with clinician goals, and be measurable/demonstrable both before and after any variation reduction changes are implemented [23].

The healthcare organization must decide if addressing the factors involved in the unwarranted variation is within current organizational capacity. For example, should the factors involved be predominately consumer/patient, an organization may choose to develop a consumer-based education and training program, or develop consumer targeted infrastructure such as a patient portal. Alternatively, the organization may deem consumer factors as outside of their control, not financially viable or simply not aligned with core goals and instead elect to abandon this issue and with the use of the "Not viable" feedback loop, adjust and select another area of variation with a higher priority to address (Figure 1).

\section{Data collection/synthesis}

Data collection and synthesis is undertaken with data collected from a variety of means and systems. IT advancements have made improvements in the availability, collection, and manipulation of these data, often allowing near real-time analysis of key performance indicators via electronic dashboards and summaries.

These include:

- IT systems, inc. EMR, CIS, payroll, billing etc.

- Key performance indicators (KPIs)

- Meetings/ discussions

- Surveys/polls, patient reported experience/outcome measures (PREMs \& PROMs)

- Anecdotal evidence

\section{Degree/type of variation}

Process input factor determination is also influenced by the type of variation that is identified, this may be variation from a level/standard, or variation around a level/standard.

Variation from a level may be clinician adherence to an existing health service guideline, such as how closely the clinician adheres to the guidelines of practice or whether clinicians are meeting gold standard best practice guidelines.

Variation around a level refers to the difference in practice outcomes after implementing a clinical guideline. This may be the implementation of a new best-practice guideline or existing guideline revisions. There are occasions where both are addressed with a single intervention. EMRs may be introduced to both implement a standard of care and achieve greater consistency in clinical practice [24].

\subsection{Team development}

(Figure 1-III)

The core team responsible for the development of the solution was comprised of a multi-disciplinary group of resources with representation from all relevant disciplines including practitioner/clinicians, champions, management, administration, financial, IT, analytics and potentially consumer/patients, working with a commitment to the quality improvement initiative [25].

With a bottom-up approach and a wide variety of stakeholders from all levels of the organization, the team's understanding, concerns and solution development will be enhanced through a comprehensive 
understanding of the issues from multiple perspectives [23].

Potential core team members include:

- Administration - providing administrative and project management and support activities.

- Practitioners - active clinicians who are providing the healthcare service, doctors, nurses, allied health etc.

- Champions - a leading clinician from the field with a thorough understanding of the issue and the solution who is able to convey the message to colleagues.

- Management - representatives of organizational management, operations management department head, chief information officer (CIO), chief clinical information officer (CCIO) etc.

- Analytics - data analytics representation for data set analysis and requirements.

Non-core members include:

- IT - technical representation offering advice throughout the project lifecycle.

- Finance - a member of the finance/accounting team able to understand the parameters of the work.

- Consumers - as required, consumer representatives to provide insight into the patient perspective of proposed solutions.

\subsection{Solution development/implementation}

(Figure 1-IV)

The intervention developed may include pillars of technical, change management, practice and underlying clinical theory and should be targeted to the process input factor(s) identified earlier in the process.

Figure 2: Unwarranted variation reduction intervention pillars

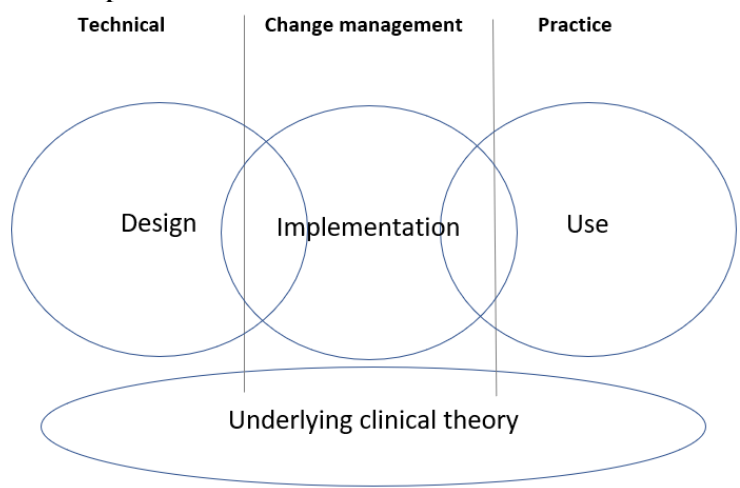

\section{Technical}

The technical dimension involves identifying a system design that can help achieve the right level of variation (i.e. through the guidance it provides to users or the constraints or controls that it enforces). Technical priorities involved in the development of an intervention include access to adequate resources (including technical staff, infrastructure, and information) as well as clinician access for testing and development.

To address this dimension, health services should seek to use an appropriately designed core health system that can provide the ability for continuation of patient care across the entire patent journey not just at a point in time. The technical system should also allow for adequate local customization to meet the requirements of the individual department, facility, or health service. This may include the need for local resources to provide development and customization.

\section{Change management}

The change management process.is critical to the success of an intervention. A clear vision and communication strategy is key to ensuring the alignment of all stakeholders. This includes stakeholder education and training, ongoing support and the measurement and monitoring of performance[26].

There is potential that the use of an intervention invokes behavioral and cultural change which takes both time and effort to achieve and maintain within any organization.

\section{Practice}

An intervention used by clinicians may be influenced by the clinicians' level of knowledge, skill set or style of practice. Monitoring of the frontline clinical practice may identify any issues with clinician use (or avoidance of use) of the intervention.

\section{Underlying clinical theory}

Variation-reducing interventions should be based on underlying clinical theory - a clinically-defensible rationale for why the reduction in variation is appropriate. One approach to identifying the appropriate theory is to examine the guidelines, standards, care plans, or treatment plans that are best practice or industry standard in that domain. These are often developed by peak bodies and customized for local conditions. While these documents do not always explicate their underlying theory, it can often be uncovered through analyzing their composition and exploring their background materials.

There are numerous ways available to modify the behavior and clinical workflow in order to reduce unwarranted clinical variation, via process control 
efforts (e.g., clinical guidelines and pathways), and process design and development efforts [3, 4].

This may be achieved via clinician training, guidelines/standards or certification through peak organizations, knowledgebases, masterclasses, and the provision of accessible information, or via system access controls forcing or limiting what is allowable by the clinician within the CIS/EMR (e.g. ordering, access, scheduling etc.).

\subsection{Outcome monitoring and review (Figure 1-V)}

The outcome monitoring and review element of the model has a dual role, providing an ongoing monitoring function as well as a feedback loop for solution adjustment. Ongoing periodic monitoring, reporting and review of clinical outcomes is essential to ensure that variation is minimized in the long-term.

Outcome monitoring and review also provides a feedback function as new information comes to light while moving through the model. The viability and appropriateness of the solution may be constantly monitored and potentially adjusted.

\subsection{Solution adjustment}

(Figure 1-VI)

The model cycle is closed with solution adjustment and revision. This may include redefining target outcomes, modifications to element(s) of the solution in any of the pillar(s) of technical, change management, practice, and underlying clinical theory, from minor refinements through to the development of completely new interventions.

\subsection{Organizational enablement (LHS) (Figure 1-VII)}

In order for a sustained level of success for organizational QI initiatives, an ethos of continued improvement needs to underpin the healthcare organizational structure. Learning health systems (LHS) have been developed around the core values of personfocused, privacy, inclusiveness, transparency, accessibility, adaptability, governance, cooperative and participatory leadership, scientific integrity and value [27]. A long-term committed approach is required to position/reposition a healthcare organization as an LHS.

The PDSA cycle should be considered within the greater LHS context and within the learning cycle (Figure 1). Compatibility between the PDSA QI cycle and the LHS learning cycle has been previously identified with both cycles containing complementary components and goals [28]. The learning cycle comprises similar elements of data gathering/analysis, feedback and implementing change and scale [29].

The stewardship and governance of LHSs is a task that becomes more difficult as systems scale to national or international levels involving various parties with fundamental differences in legal and ethical positions. Data considerations including sources, collection, standards, formats, quality, interoperability, security and storage, privacy and policies are all factors that the LHS my need to address [30].

These systems bring with them a change in demands for real-time information and opportunities to utilize technologies such as EMRs to disseminate clinical knowledge at a more rapid rate than the existing peer-reviewed journal articles process.

\section{Limitations and future research}

The LHS, and unwarranted clinical variation reduction process models revolve around the ability of the organization to develop solutions iteratively. This may not be the case when developing interventions within the real-world of healthcare budget cycles which are often underpinned with time-limited specific funding. Funding via periodic budgets, grants, sponsorships etc. makes the implementation of any solution potentially a one-off task [31]. In these cases, the iteration of a solution design may be replaced with the development of lessons learned to be utilized in future innovations.

As the proposed model is multifaceted, identification of and control for, local variables may be required.

This research is focused on the healthcare and clinical informatics setting, which would be beneficial to extend to other industries. There are potentially additional learnings around quality improvement that may be observed from other industries that can be applied back to the healthcare realm.

Further validation both conceptually and empirically is to be undertaken as the next phase of this research.

While there has been some pivotal work undertaken exploring clinical practice variation, continued research on these variation reducing initiatives is essential to ensure the most effective implementation and utilization of these systems [32, 33].

\section{Conclusion}

Healthcare organizations attempting to address unwarranted clinical variation face a complex task, filled with potential trade-offs between separate and 
often competing elements of the quadruple aim of healthcare, to improve patient care, population health, cost of care, and clinician experience. To date, most organizations have focused on meeting one or two of the quadruple aims, typically cost/efficiency and/or patient care outcomes [6].

By striving to become learning organizations and closing the loop with consistent outcome monitoring and adjustment (Figure 1), combined with the latest digital health technologies and collaborative methodologies, healthcare organizations stand the

\section{References}

[1] R. Sikka, J. M. Morath, and L. Leape, "The quadruple aim: care, health, cost and meaning in work," Editorial 2015.

[2] D. M. Berwick, T. W. Nolan, and J. Whittington, "The triple aim: care, health, and cost," (in eng), Health Aff (Millwood), vol. 27, no. 3, pp. 759-69, May-Jun 2008, doi: $10.1377 /$ hlthaff.27.3.759.

[3] C. P. McLaughlin, "Why variation reduction is not everything: a new paradigm for service operations," International Journal of Service Industry Management, 1996.

[4] C. McLaughlin and S. Johnson, "Inherent variability in service operations: identification, measurement and implications," Services Management: New Directions and Perspectives, Cassell, London, pp. 226-9, 1995.

[5] L. M. Prevedello, A. S. Raja, I. K. Ip, A. Sodickson, and R. Khorasani, "Does clinical decision support reduce unwarranted variation in Yield of CT pulmonary angiogram?," (in English), American Journal of Medicine, Article vol. 126, no. 11, pp. 975-981, 2013, doi: 10.1016/j.amjmed.2013.04.018.

[6] T. Hodgson, A. Burton-Jones, R. Donovan, and C. Sullivan, "The role of EMRs in reducing unwarranted variation in acute health care processes: a systematic review," Under review, 2021.

[7] G. R. Cohen, C. P. Friedman, A. M. Ryan, C. R. Richardson, and J. Adler-Milstein, "Variation in physicians' electronic health record documentation and potential patient harm from that variation," Journal of general internal medicine, vol. 34, no. 11, pp. 23552367, 2019.

[8] M. J. Taylor, C. McNicholas, C. Nicolay, A. Darzi, D. Bell, and J. E. Reed, "Systematic review of the application of the plan-do-study-act method to improve quality in healthcare," BMJ quality \& safety, vol. 23, no. 4, pp. 290-298, 2014.

[9] Healthcare Quality Improvment Partnership, "Best practice in clinical audit," Healthcare Quality Improvement Partnership Ltd., 2020.

[10] J. Thor et al., "Application of statistical process control in healthcare improvement: systematic review," (in eng), Qual Saf Health Care, vol. 16, no. 5, pp. 387-399, 2007, doi: 10.1136/qshc.2006.022194. greatest chance to reduce unwarranted clinical variation and optimize healthcare outcomes.

\section{Acknowledgements}

This research was funded via an Australian
Research Council Linkage Projects grant
(LP170101154).

[11] P. Christoff, "Running PDSA cycles," Current problems in pediatric and adolescent health care, vol. 48, no. 8, pp. 198-201, 2018.

[12] R. Wirth and J. Hipp, "CRISP-DM: Towards a standard process model for data mining," in Proceedings of the 4th international conference on the practical applications of knowledge discovery and data mining, 2000, vol. 1: Springer-Verlag London, UK.

[13] G. D. Cleghorn and L. A. Headrick, "The PDSA Cycle at the Core of Learning in Health Professions Education," The Joint Commission Journal on Quality Improvement, vol. 22, no. 3, pp. 206-212, 1996/03/01/ 1996, doi: https://doi.org/10.1016/S10703241(16)30223-1.

[14] W. A. Shewhart and W. E. Deming, Statistical method from the viewpoint of quality control. Courier Corporation, 1986.

[15] W. E. Deming, Out of the Crisis. MIT press, 2018.

[16] ACSQHC. "Healthcare Variation | Australian Commission on Safety and Quality in Health Care." Online. https://www.safetyandquality.gov.au/ourwork/healthcare-variation (accessed 26/11/2020.

[17] H. Asri, H. Mousannif, H. Al Moatassime, and T. Noel, "Big data in healthcare: challenges and opportunities," in 2015 International Conference on Cloud Technologies and Applications (CloudTech), 2015: IEEE, pp. 1-7.

[18] T. J. Hannan, "Variation in health care - the roles of the electronic medical record," International Journal of Medical Informatics, vol. 54, no. 2, pp. 127-136, 1999.

[19] U. Guo, L. Chen, and P. H. Mehta, "Electronic health record innovations: Helping physicians-One less click at a time," Health Information Management Journal, vol. 46, no. 3, pp. 140-144, 2017.

[20] A. Duclos and N. Voirin, "The p-control chart: a tool for care improvement," International Journal for Quality in Health Care, vol. 22, no. 5, pp. 402-407, 2010.

[21] V. Cao et al., "Patient-centered structured interdisciplinary bedside rounds in the medical ICU," Critical care medicine, vol. 46, no. 1, pp. 85-92, 2018.

[22] A. S. Detsky, "Regional variation in medical care," 1995.

[23] C. Castano, M. Love, R. Den Ouden, L. v. D. Neelen, Michael, and A. M. Giusto, "Working in Concert: A How-To Guide to Reducing Unwarranted Variation in Care."

[24] K. K. Ganju, H. Atasoy, J. McCullough, and B. Greenwood, "The role of decision support systems in 
attenuating racial biases in healthcare delivery," Management Science, vol. 66, no. 11, pp. 5171-5181, 2020.

[25] M. A. Rosen et al., "Teamwork in healthcare: Key discoveries enabling safer, high-quality care," (in eng), Am Psychol, vol. 73, no. 4, pp. 433-450, May-Jun 2018, doi: 10.1037/amp0000298.

[26] R. Al-Abri, "Managing change in healthcare," Oman medical journal, vol. 22, no. 3, p. 9, 2007.

[27] L. H. Community, "Endorsers of the core values underlying a national-scale person-centered continuous Learning Health System (LHS)."

[28] T. J. Foley and L. Vale, "What role for learning health systems in quality improvement within healthcare providers?," Learning Health Systems, vol. 1, no. 4, p. e10025, 2017.

[29] C. P. Friedman and J. Macy Jr, "Toward complete \& sustainable learning systems," University of Michigan, 2014.

[30] J. M. McGinnis, B. Powers, and C. Grossmann, "Digital infrastructure for the learning health system: the foundation for continuous improvement in health and health care: workshop series summary," 2011.

[31] K. Walsh, "Managing a Budget in Healthcare Professional Education," (in eng), Ann Med Health Sci Res, vol. 6, no. 2, pp. 71-73, Mar-Apr 2016, doi: $10.4103 / 2141-9248.181841$.

[32] M. J. Ott and G. H. Olsen, "Impact of quality assessment on clinical practice, Intermountain healthcare," in Quality Spine Care: Springer, 2019, pp. 301-313.

[33] T. K. Colicchio et al., "Looking behind the curtain: identifying factors contributing to changes on care outcomes during a large commercial EHR implementation," eGEMs, vol. 7, no. 1, 2019. 